\title{
Non-Rayleigh scattering behavior for anisotropic Rayleigh particles
}

\author{
Y. X. Ni, ${ }^{1}$ L. Gao, ${ }^{1, *}$ A. E. Miroshnichenko, ${ }^{2,4}$ and C. W. Qiu ${ }^{3,5}$ \\ ${ }^{1}$ Jiangsu Key Laboratory of Thin Films, Department of Physics, Soochow University, 1 Shizi Street, Suzhou 215006, China \\ ${ }^{2}$ Nonlinear Physics Centre, Australian National University, Canberra, Australian Capital Territory 0200, Australia \\ ${ }^{3}$ Department of Electrical and Computer Engineering, National University of Singapore, 4 Engineering Drive 3, \\ Singapore 117576, Singapore \\ ${ }^{4}$ e-mail: aem124@physics.anu.edu.au \\ ${ }^{5}$ e-mail: eleqc@nus.edu.sg \\ *Corresponding author: leigao@suda.edu.cn
}

Received April 30, 2012; revised June 26, 2012; accepted July 3, 2012;

posted July 5, 2012 (Doc. ID 167735); published August 8, 2012

\begin{abstract}
Derived from the light scattering by a radially anisotropic sphere, unusual scattering behavior is exhibited, which breaks the Rayleigh law (scattering efficiency $Q_{\text {sca }} \sim q^{4}$ as $q \rightarrow 0$, where $q$ is the size parameter). Under certain conditions, we demonstrate an asymptotical relation between $Q_{\mathrm{sca}}$ and $q$, i.e., $Q_{\mathrm{sca}}=F q^{8}$, which is not realizable for isotropic particles in Rayleigh regime. Moreover, suitable adjustment of the anisotropic parameters can further suppress the coefficient $F$, resulting in enhanced transparency of the anisotropic particle. @ 2012 Optical Society of America
\end{abstract}

OCIS codes: $\quad 290.4020,290.5870,290.5850,160.1190$.

Light scattering by a small obstacle is one of the most fundamental problems in classical electrodynamics [1-8]. The pioneering researcher is Rayleigh [1], who studied the elastic scattering of light by spherical particles in the small particle limit. For a homogeneous sphere with arbitrary size, the full-wave electromagnetic theory was established by Mie [2]. For instance, the scattering cross section can be written in terms of the Mie scattering coefficients $a_{n}$ and $b_{n}$ [3,4]. In the Rayleigh limit with both $q=(2 \pi a / \lambda) \ll 1(a$ is the radius of the sphere and $\lambda$ is the incident wavelength) and $m q \ll 1$ ( $m$ is the refractive index of the sphere relative to the surrounding medium), the scattering of electromagnetic radiation is dominated by electric and magnetic dipoles, and the scattering efficiency $Q_{\mathrm{sca}}$ is found to be proportional to $q^{4}$ in the case that the sphere is made of conventional materials $(\varepsilon>0$ and $\mu>0$ ). In addition, for small particles with low dissipation rates, light scattering is unusual [5] and Fano resonances are observed [6,7]. Motivated by both experimental and theoretical developments in the field of metamaterials with simultaneous negative permittivity and permeability $[\underline{9}, 10]$, non-Rayleigh limits of the small particles made of metamaterials were demonstrated such as $Q_{\text {sca }} \sim 1 / q^{2}$ or $Q_{\text {sca }} \sim$ const under certain conditions $[11,12]$. Recently, light scattering by nanoparticles with radial anisotropy received much attention [13-17].

In this letter, we adopt the full-wave electromagnetic theory to study light scattering by small particles with radial anisotropy. Especially, we analytically study the scattering efficiency in the Rayleigh limit, and the unusual behavior of $Q_{\mathrm{sca}}$ as a function of the size parameter $q$ is predicted $Q_{\text {sca }} \sim q^{8}$ instead of the Rayleigh law $Q_{\text {sca }} \sim q^{4}$. As a consequence, with decreasing $q$, the scattering efficiency by anisotropic particles may vanish much faster than the one by the isotropic particles. Moreover, for the particles with radial anisotropy, suitable adjustment of the permittivity and permeability can result in much smaller scattering efficiency and is helpful to realize near-transparency.
Let us consider the scattering of light by a spherical particle with radial anisotropy of radius $a$, surrounded by free space with permittivity $\varepsilon_{0}$ and permeability $\mu_{0}$. Radial anisotropy means that the physical parameters such as the permittivity and permeability of the particles differ when measured along the radial and tangential directions to the surface. The relative permittivity and permeability tensors are written in the spherical coordinates as $\overline{\bar{\varepsilon}}=\left(\varepsilon_{r} \hat{r} \hat{r}+\varepsilon_{t} \hat{\theta} \hat{\theta}+\varepsilon_{t} \hat{\phi} \hat{\phi}\right), \overline{\bar{\mu}}=\left(\mu_{r} \hat{r} \hat{r}+\mu_{t} \hat{\theta} \hat{\theta}+\mu_{t} \hat{\phi} \hat{\phi}\right)$. For simplicity, we assume the polarized incident wave with unit amplitude: $E_{i}=\hat{e}_{x} e^{i k_{0} z}$, where $k_{0}=\omega \sqrt{\varepsilon_{0} \mu_{0}}$. Here, we generalize the Mie scattering theory [ $[\underline{3}, \underline{4}]$ to study light scattering by the spherical particle with radial anisotropy. After some algebraic manipulations, we obtain the $n$th TM scattering coefficients $a_{n}[\underline{13}, \underline{14}]$,

$$
a_{n}=\frac{\sqrt{\varepsilon_{t}} \psi_{n}^{\prime}(q) \psi_{\nu_{n}}(m q)-\sqrt{\mu_{t}} \psi_{n}(q) \psi_{\nu_{n}}^{\prime}(m q)}{\sqrt{\varepsilon_{t}} \xi_{n}^{\prime}(q) \psi_{\nu_{n}}(m q)-\sqrt{\mu_{t}} \xi_{n}(q) \psi_{\nu_{n}}^{\prime}(m q)},
$$

where $m=\sqrt{\varepsilon_{t}} \sqrt{\mu_{t}}$ is the refractive index of the sphere relative to vacuum, $\psi_{i}(x)$ and $\xi_{i}(x)$ are the Ricatti-Bessel functions, and the primes indicate differentiation with respect to the entire argument. Note that $\nu_{n}=$ $\sqrt{n(n+1) \varepsilon_{t} / \varepsilon_{r}+1 / 4}-\frac{1}{2}$. For the isotropic particle with $\varepsilon_{r}=\varepsilon_{t}$, we have $\nu_{n}=n$, Eq. (1) is just the Mie coefficients. Incidentally, the $n$th $\mathrm{TE}$ scattering coefficients $b_{n}$ can be similarly obtained by $\varepsilon_{i} \leftrightarrow \mu_{i}(i=t$ or $r)$ and $\nu_{n} \rightarrow \gamma_{n}\left(\gamma_{n}=\sqrt{n(n+1) \mu_{t} / \mu_{r}+1 / 4}-\frac{1}{2}\right)$ in Eq. (1).

The scattering efficiency is defined by $[\underline{3}, \underline{4}]$

$$
Q_{\mathrm{sca}}=\frac{2}{q^{2}} \sum_{n=1}^{\infty}(2 n+1)\left(\left|a_{n}\right|^{2}+\left|b_{n}\right|^{2}\right) .
$$

Here we focus on what happens in the Rayleigh limit (both $q \ll 1$ and $m q \ll 1$ ), where the first-order coefficients $a_{1}$ and $b_{1}$ contribute to the scattering efficiency mainly. 
The Riccati-Bessel functions for noninteger orders $\nu_{n}$ are $\psi_{\nu_{n}}(x)=\sqrt{\frac{\pi x}{2}} \sum_{k=0}^{\infty} \frac{(-1)^{k}}{k ! \Gamma\left(\nu_{n}+k+3 / 2\right)}\left(\frac{x}{2}\right)^{2 k+\nu_{n}+1 / 2}$, with the Euler gamma function $\Gamma(\cdots)$. As a consequence, $a_{1}$ can be approximately written as,

$$
\begin{aligned}
a_{1}= & -\frac{i\left(2 C_{1} \sqrt{\varepsilon_{t}}-D_{1} \sqrt{\mu_{t}}\right)}{3\left(C_{1} \sqrt{\varepsilon_{t}}+D_{1} \sqrt{\mu_{t}}\right)} q^{3} \\
& +\frac{i\left(4 C_{1}+20 C_{2}\right) \sqrt{\varepsilon_{t}}-i\left(D_{1}+10 D_{2}\right) \sqrt{\mu_{t}}}{30\left(C_{1} \sqrt{\varepsilon_{t}}+D_{1} \sqrt{\mu_{t}}\right)} q^{5},
\end{aligned}
$$

with the parameters $C_{j}=(m / 2)^{\nu_{1}+2 j-1} \sqrt{\pi} / \Gamma\left[\nu_{1}+(2 j+\right.$ 1)/2] and $D_{j}=(m / 2)^{\nu_{1}+2 j-2} \sqrt{\pi}\left(\nu_{1}+2 j-1\right) /\left\{\Gamma\left[\nu_{1}+(2 j-\right.\right.$ 1) $\left./ 2]\left(2 \nu_{1}+2 j-1\right)\right\}(j=1,2)$.

For an isotropic small sphere containing conventional materials, with which the permittivity and permeability are not equal to 1 , both $a_{1}$ and $b_{1}$ are found to be proportional to $q^{3}$, then the scattering efficiency is governed by the well-known Rayleigh law. Here, for anisotropic spheres, we find that the scattering efficiency can exhibit another unusual dependence of $q$. For instance, the first term in Eq. (3) will vanish for $\varepsilon_{t}=\left(\varepsilon_{r}+1\right) /\left(2 \varepsilon_{r}\right)$. As a result, $a_{1}$ admits the form

$$
a_{1}=\frac{i}{45}\left(1-\frac{5 \mu_{t}}{4 \varepsilon_{t}+1}\right) q^{5}
$$

Similarly, when the components of the permeability tensor satisfy the relation $\mu_{t}=\left(\mu_{r}+1\right) /\left(2 \mu_{r}\right), b_{1}$ is simplified as $b_{1}=i q^{5}\left[1-5 \varepsilon_{t} /\left(4 \mu_{t}+1\right)\right] / 45$. Since the firstorder scattering coefficients $\left(a_{1}\right.$ and $\left.b_{1}\right)$ are proportional to $q^{5}$, the contributions of the second-order scattering coefficients $\left(a_{2}\right.$ and $\left.b_{2}\right)$ should not be neglected. We have

$$
a_{2}=-\frac{i}{45} \frac{3 \varepsilon_{t}-\left(\sqrt{6 \varepsilon_{t}\left(2 \varepsilon_{t}-1\right)+1 / 4}+1 / 2\right)}{2 \varepsilon_{t}+\left(\sqrt{6 \varepsilon_{t}\left(2 \varepsilon_{t}-1\right)+1 / 4}+1 / 2\right)} q^{5} .
$$

Again, the expression for $b_{2}$ follows from Eq. (5) with $\varepsilon_{t} \leftrightarrow \mu_{t}$.

Substituting these coefficients into Eq. (2) results in

$$
\begin{aligned}
Q_{\mathrm{sca}} & \approx \frac{2 q^{8}}{675}\left[\left(1-\frac{5 \mu_{t}}{4 \varepsilon_{t}+1}\right)^{2}+\left(1-\frac{5 \varepsilon_{t}}{4 \mu_{t}+1}\right)^{2}\right] \\
+ & \frac{2 q^{8}}{405} \sum_{g=\varepsilon}^{\mu}\left(\frac{6 g_{t}-\sqrt{24 g_{t}\left(2 g_{t}-1\right)+1}-1}{4 g_{t}+\sqrt{24 g_{t}\left(2 g_{t}-1\right)+1}+1}\right)^{2} .
\end{aligned}
$$

Hence, for anisotropic particles, under proper conditions, we find analytically $Q_{\text {sca }} \sim q^{8}$ instead of the Rayleigh law $Q_{\text {sca }} \sim q^{4}$. Actually, this change results from the introduction of additional anisotropic parameters. Tuning these parameters, one may make the leading terms zero in the expansion of $Q_{\mathrm{sca}}$ in powers of $q$, so that the subleading terms begin to play a dominant role. In addition, as $q \rightarrow 0$, it is possible to realize faster vanishing of the scattering efficiency with the anisotropic particles, resulting in better transparency in comparison with isotropic particles.

To verify our analytical theory, we numerically calculate the scattering efficiency with the full-wave electromagnetic theory based on Eqs. (1) and (2), see Fig. 1. We find that for ordinary anisotropic physical parameters $\left(\varepsilon_{t}=3, \quad \varepsilon_{r}=1, \mu_{t}=5\right.$, and $\left.\mu_{r}=1\right)$, the scattering efficiency obeys the Rayleigh law. However, $\varepsilon_{t}=3$, $\varepsilon_{r}=1 / 5, \mu_{t}=5$, and $\mu_{r}=1 / 9$, which satisfy the relations $\varepsilon_{t}=\left(\varepsilon_{r}+1\right) /\left(2 \varepsilon_{r}\right)$ and $\mu_{t}=\left(\mu_{r}+1\right) /\left(2 \mu_{r}\right)$, and one observes unusual scattering asymptotical behavior with $Q_{\text {sca }} \sim q^{8}$. The full-wave numerical results (the solid line) are in good agreement with our analytical derivations with Eq. (ㅁ) as expected. Here we would like to mention that for nonmagnetic anisotropic particles $\left(\mu_{t}=\mu_{r}=1\right)$, such unusual behavior is still possible to obtain (see the dotted line). This is very important for possible applications.

Generally, as $q \rightarrow 0, Q_{\text {sca }}$ as a function of $q$ always has a form $Q_{\text {sca }}=F q^{\sigma}$, where both the coefficient $F$ and the exponent $\sigma$ depend on the physical parameters. For $g_{t}=$ $\left(g_{r}+1\right) /\left(2 g_{r}\right)(g=\varepsilon$ or $\mu)$, we have predicted the fast vanishing of the scattering efficiency with $\sigma=8$ in comparison with Rayleigh's prediction with $\sigma=4$. It is natural to ask the question whether one can further decrease the scattering efficiency through the decrease of coefficient $F$. In Fig. 2, we show the unusual suppressed scattering efficiency versus size parameter under our conditions. We find that all lines take the same slope with $\sigma=8$, but have different scattering magnitudes. This is due to the fact that the coefficient $F$ can be tuned by adjusting the permittivity $\varepsilon_{t}$ for a given $\mu_{t}$ (or $\mu_{r}$ ), and it achieves a minimal value for $\varepsilon_{t}=\mu_{t} \quad(F=0$ for $\left.\varepsilon_{t}=\varepsilon_{r}=\mu_{t}=\mu_{r}=1\right)$. In our case, with increasing $\varepsilon_{t}$, $F$ decreases initially, and goes through a minimum at an optimal $\varepsilon_{t}=3$, then increases (see the inset). Thus, one may control the rate of the suppression of $Q_{\text {sca }}$ through tuning $\varepsilon_{t}$.

In the end, we would like to examine the origin of changes in the asymptotic behavior of $Q_{\text {sca. }}$. In the Rayleigh limit, the anisotropic particle can be regarded

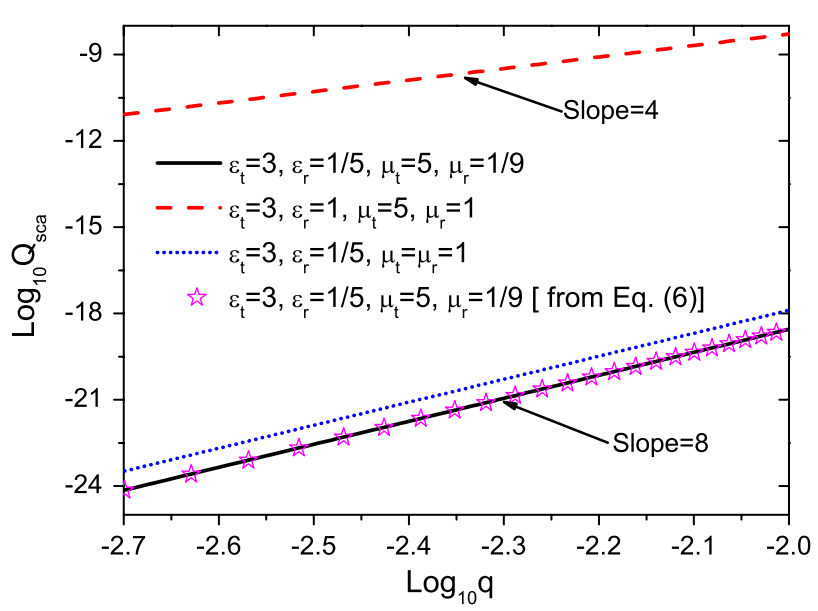

Fig. 1. (Color online) Log-log plot of the scattering efficiency against the size parameters. The lines are calculated via fullwave electromagnetic theory. The magenta star is calculated with Eq. (ㅁ). 


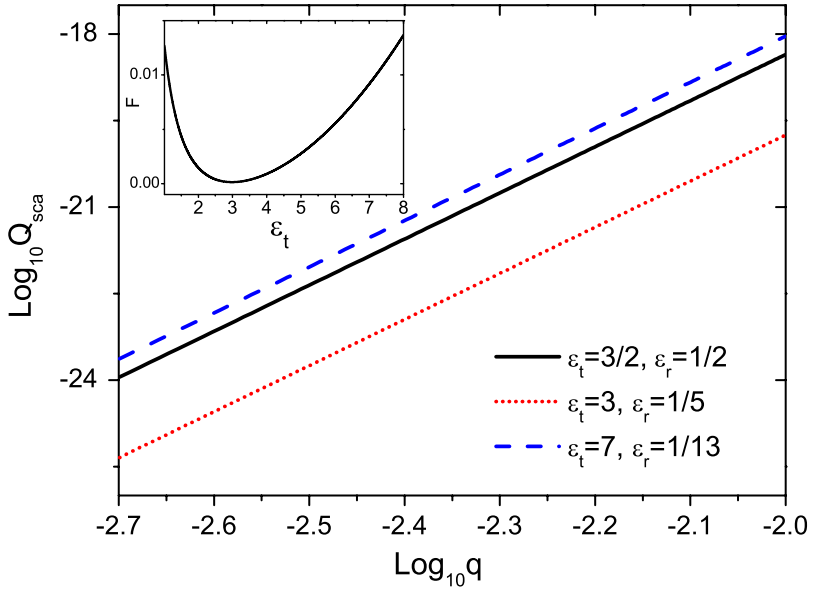

Fig. 2. (Color online) $Q_{\text {sca }}$ versus $q$ and $F$ versus $\varepsilon_{t}$ (the inset) for $\mu_{t}=3$ and $\mu_{r}=1 / 5$.

as the isotropic one with the equivalent permittivity $\varepsilon_{e}=\varepsilon_{r} \nu_{1}$ and the equivalent permeability $\mu_{e}=\mu_{r} \gamma_{1}$ $[\underline{14}, 18]$. For $g_{t}=\left(g_{r}+1\right) /\left(2 g_{r}\right)$, it is easy to obtain $\varepsilon_{e}=1$ and $\mu_{e}=1$. In other words, such an anisotropic spherical scatterer behaves as an isotropic sphere made of vacuum [19]. As a consequence, one may observe the unusual suppressed scattering efficiency. In addition, one can check the electric field distribution, as shown in Fig. 3. When the conditions $g_{t}=\left(g_{r}+1\right) /\left(2 g_{r}\right)$ are satisfied, the scattering field is quite small (see Fig. 3(a)), otherwise the scattering field cannot be ignored [see Fig. 3(b)].

In summary, we study light scattering by small spheres with radial anisotropy via the full-wave electromagnetic theory. We predict the unusual dependence of the scattering efficiency on small $q$ such as $Q_{\text {sca }}=F q^{8}$ for $\varepsilon_{t}=$ $\left(\varepsilon_{r}+1\right) /\left(2 \varepsilon_{r}\right)$ and $\mu_{t}=\left(\mu_{r}+1\right) /\left(2 \mu_{r}\right)$, and one may obtain low scattering efficiency by small particles with radial anisotropy. Such unusual asymptotic behavior is absent for the isotropic spheres, while this is novel for the anisotropic spheres. Moreover, the coefficient $F$ can be further decreased by the adjustment of the physical parameters. Therefore, it is possible to yield much lower scattering efficiency, and hence better transparency with anisotropic particles.

This work was supported by the National Natural Science Foundation of China (NSFC, No. 11074183), the National Basic Research Program (No. 2012CB921 501), the Key Project in National Science Foundation (NSF) of Jiangsu Education Committee (No. 10KJA 140044), and Priority Academic Program Development



Fig. 3. (Color online) Contour plot of electric field enhancement $\left|E / E_{0}\right|$ in the $x / a-z / a$ plane for $\mu_{t}=\mu_{r}=1$, $q=0.002$. Other parameters are: (a) $\varepsilon_{t}=3, \varepsilon_{r}=1 / 5$; (b) $\varepsilon_{t}=3, \varepsilon_{r}=2$. Note that the area outside the sphere includes the scattering field only.

(PAPD) of Jiangsu Higher Education Institutions. The work of A. E. M. was supported by the Australian Research Council through the Future Fellowship program.

\section{References}

1. Lord Rayleigh, Philos. Mag. 41, 107 (1871).

2. G. Mie, Ann. Phys. 25, 377 (1908).

3. C. F. Bohren and D. R. Huffman, Absorption and Scattering of Light by Small Particles (Wiley, 1998).

4. M. Born and E. Wolf, Principles of Optics: Electromagnetic Theory of Propagation, Interference and Diffraction of Light, 7th (expanded) ed. (Cambridge, 1999).

5. M. I. Tribelsky and B. S. Luk'yanchuk, Phys. Rev. Lett. 97, 263902 (2006).

6. M. I. Tribelsky, S. Flach, A. E. Miroshnichenko, A. V. Gorbach, and Y. S. Kivshar, Phys. Rev. Lett. 100, 043903 (2008).

7. A. E. Miroshnichenko, S. Flach, and Y. S. Kivshar, Rev. Mod. Phys. 82, 2257 (2010).

8. V. B. Il'in and V. G. Farafonov, Opt. Lett. 36, 4080 (2011)

9. M. C. K. Wiltshire, Science 292, 60 (2001).

10. R. A. Shelby, D. R. Smith, and S. Schultz, Science 292, 77 (2001).

11. Z. Liu, Z. F. Lin, and S. T. Chui, Phys. Rev. E 69, 016609 (2004)

12. A. E. Miroshnichenko, Phys. Rev. A 80, 013808 (2009).

13. B. S. Luk'yanchuk and C. W. Qiu, Appl. Phys. A 92, 773 (2008).

14. L. Gao, T. H. Fung, K. W. Yu, and C. W. Qiu, Phys. Rev. E 78, 046609 (2008).

15. C. W. Qiu, L. Gao, J. D. Joannopoulos, and M. Soljacic, Laser Photon. Rev. 4, 268 (2010).

16. X. F. Fan, Z. X. Shen, and B. Luk'yanchuk, Opt. Express 18, 24868 (2010).

17. Z. J. Li, Z. S. Wu, and Q. C. Shang, Opt. Express 19, 16044 (2011)

18. Y. X. Ni, D. L. Gao, Z. F. Sang, L. Gao, and C. W. Qiu, Appl. Phys. A 102, 673 (2011).

19. H. Chew and M. Kerker, J. Opt. Soc. Am. 66, 445 (1976). 